\title{
EVALUATION OF HERNIORRHAPHY FOR PARA - UMBILICAL HERNIA ASSOCIATED WITH LAPAROSCOPIC CHOLECYSTECTOMY
}

By

\section{Ahmed Radwan Ali Ahmed, Selim Saied Abd El-Rahman El-Nemr and Mahmoud Abd El-Hady Abd El-Aziz}

Department of General Surgery, Faculty of Medicine, Al-Azhar University

Corresponding author: Ahmed Radwan Ali Ahmed,

E-mail: ahmed_radwan55@gmail.com

\begin{abstract}
Background: Para-umbilical hernia repair (PUHR) combined with LC result in longer operating time, longer anesthesia, and risk of increased blood loss. But it has the advantages of single hospital stay, single anesthesia exposure, less post-operative pain and morbidity, early return to work, better cosmesis and more convenient, efficacy and cost effective for the patient.
\end{abstract}

Objective: To evaluate the selected repair methods for para-umbilical hernias that already exist or are encountered incidentally and to present data regarding potential problems that may occur during laparoscopic cholecystectomy.

Patients and Methods: During period of January 2017 to January 2019, laparoscopic cholecystectomy was attempted in 25 patients suffering from chronic calcular cholecystitis accompanied by para-umbilical hernia, patients were assigned to two groups according to occurrence of recurrence. A retrospective analysis of parameters, including patient demographics, laboratory values, radiologic data, and intraoperative findings, was performed.

Results: The operating time, postoperative pain, length of hospital stay, wound infection and the time needed for return to work were less in patients without recurrence than patients with recurrence. Recurrence occurred in cases with BMI 35.5 \pm 8.08 , patient with comorbidities (DM and hypertension) and size of the defect $2.4 \pm .07 \mathrm{~cm}$. Cases with recurrence have longer operational time and hospital stay than cases without recurrence and take more days to return to work. Cases with recurrence have also other complication such as hematoma and wound infection. There was a moderate positive correlation between BMI and both operating time and length of hospital stay. There was strong positive correlation between size of hernia and both operating time and length of hospital stay. There was a strong positive correlation between comorbidity and both operating time and length of hospital stay.

Conclusion: Para-umbilical hernia repair combined with laparoscopic cholecystectomy resulted in longer operating time, longer anesthesia, and risk of increased blood loss. But it has the advantages of single hospital stay, single anesthesia exposure, less postoperative pain and morbidity, early return to work, better cosmesis and more convenient, efficacy and cost effective for the patient. However, the outcomes of the para-umbilical defect repair with mesh after laparoscopic surgeries appeared to be better for either obese or non-obese patients than primary suture techniques in recurrence rates.

Keyword: Hernia repair, Laparoscopic cholecystectomy, Para-umbilical hernia. 


\section{INTRODUCTION}

Laparoscopic surgery has passed through different stages of evolution to reach its present status where it has earned the title of "Gold Standard" treatment for various surgical problems. The initial period of the learning curve has now been shortened substantially, and most centers are imparting training to budding surgeons all over the world (Malik, 2011).

Para-umbilical hernia (PUH) is mainly due to a defect in the line a alba. Hernia sac usually consists of fat tissues of peritoneal cavity, small intestine and omentum (Martis et al., 2011).

Paraumbilical hernias are more common in female than male. Factors related with occurrence and recurrence of PUH are obesity, age and multiparit .Treatment of choice is elective surgery due to chances of incarceration, obstruction and strangulation. (Farquharson et al., 2014, Kulaçoğlu, 2015 and Lanaya et al., 2018).

Previously tension free suture was used which was replaced by the usage of mesh to repair the hernia, which may reduce the rate of recurrence. Umbilical hernias (UH) comprise $6 \%$ of abdominal wall hernias in adults and it frequently accompanies cholelithiasis especially in female patients (Aslani and Brown, 2010).

With advancement in laparoscopic surgery a number of surgical procedures can be performed combined with laparoscopic cholecystectomy in a single surgery. Combined procedures provide patients with all the benefits of minimal invasive surgery and also give the benefit of single time anesthesia without adding to post-operative and hospital stay (Savita et al., 2010).

Each of the procedure performed laparoscopic ally benefits from decreased postoperative pain, early ambulation, and early return to oral feeds, early discharge and early return to work. Patient benefits from the single exposure to anesthesia, single hospital stay, and single break from work. The procedures when combined have proved equally safe and efficacious as when done singularly (Savita et al., 2010).

The aim of the present study was to evaluate the selected repair methods for para-umbilical hernias that already exist or are encountered incidentally and to present data regarding potential problems that may occur during laparoscopic cholecystectomy.

\section{PATIENTS AND METHODS}

A retrospective study during the period from January 2017 to January 2019 and included twenty-five patients at Al-Azhar University hospitals with chronic calcular cholecystitis accompanied by Paraumbilical hernia who underwent laparoscopic cholecystectomy and paraumbilical hernia repair simultaneously.

All patients had been consented for laparoscopic cholecystectomy with paraumbilical hernia repair. Patients with paraumbilical hernia were diagnosed by clinical examination in the preoperative period. Ultrasonography was performed in all patients with para-umbilical defect detected during physical examination or in those with possible hernia in order to characterize defect size and content of 
para-umbilical hernia and chronic calcular cholecystitis.

\section{Inclusion criteria:}

Any age, any sex of patients with noncomplicated chronic calcular cholecystitis associated with para-umbilical hernia with defect size less than $3 \mathrm{~cm}$.

\section{Exclusion criteria:}

Patients with associated diseases such as chronic pulmonary disease, cardiac disease, ascites, chronic renal failure, patients with strangulated para-umbilical hernia, patients with recurrent paraumbilical hernia, patients with omphalitis or periumbilical fistula and complicated cholecystitis.

The data were collected and a detailed tabulation was developed to record information on preoperative factors included age, gender, body mass index (BMI), and associated medical risk factors, i.e. diabetes mellitus, hypertension, cardiovascular disease, etc.

\section{Preoperative preparation:}

All patients had routine pre-operative laboratory investigations including complete blood count, liver and kidney function and fasting blood sugar. Diabetic patients were asked to continue their normal regimen. Electrocardiogram was done to all patients, 40 years or over. Abdominal sonography was done for assessment of gallbladder wall thickening, number of gallstones and any other intraabdominal pathology. All patients received antibiotic prophylaxis with $3 \mathrm{rd}$ generation cephalosporine intravenously during induction of anesthesia.

\section{Operative technique:}

All patients underwent elective operations under general anesthesia, laparoscopic cholecystectomy was performed using the standard four-port method, and then hernia repair was done simultaneously in the same surgery session. For the umbilical port (first port) insertion, an incision at the level of the hernia with isolation of the peritoneal sac. Through a direct cut down to the peritoneum, control of the presence of adherence and prevention of visceral injury, as in an open laparoscopy, then insertion of laparoscopic port (Hasson technique). A purse string suture is placed around the fascia and peritoneum in order to prevent excessive $\mathrm{CO} 2$ leak. Then the abdomen was insufflated to $15 \mathrm{~mm} \mathrm{Hg}$. After pneumoperitoneum was achieved, a 30-degree laparoscope was used. The 2 nd port is placed in the epigastric region. This port should be placed in a direct line to the infundibulum. This provides a good line for clip placement and avoids unnecessary torque on the instruments. A 5-mm port is then placed in the right anterior axillary line along the costal margin, between the 12th rib and the iliac crest. The final 5$\mathrm{mm}$ port is placed in the midclavicular line. Both of the 5 -mm ports should be 2 fingerbreadths below the right costal margin and should be 7 to $10 \mathrm{~cm}$ apart.

The patient can be placed in reverse Trendelenburg position to allow the duodenum, stomach, and other intraabdominal contents to fall away from the dissection field. A 5-mm locking grasper is placed through the most lateral port, and the gallbladder is retracted cephalad, toward the patient's right shoulder, A second grasper is placed through the medial 5-mm port and used to retract the infundibulum of the gallbladder laterally 
AHMED RADWAN ALI AHMED et al.,

(to the patient's right side) and inferiorly, opening up the triangle of Calot and better exposing the cystic structures. Using a Maryland dissector through the epigastric port, any adhesions between the gallbladder and the omentum, hepatic flexure, stomach, and duodenum are taken down by grasping them close to the gallbladder and peeling them down along the axis of the cystic duct. The dissection of the triangle of Calot is best performed laterally to medially, first exposing the infundibulum-cystic duct junction on the patient's right side, then medially. The cystic duct is circumferentially dissected. The cystic artery, which is usually medial and superior with the infundibulum retracted laterally, is then dissected circumferentially in similar fashion using the Maryland dissector. The cystic duct was ligated with clips applied with a 10$\mathrm{mm}$ clip applier through the epigastric port. Two clips were placed distally and one proximally on the duct. A curved or hook scissors used to divide between the most proximal and the two distal clips. The cystic artery was clipped in similar fashion and divided with the scissors, leaving two clips on the distal, retained stump. The gallbladder was dissected out of the gallbladder fossa using electrocautery. This dissection is performed from the infundibulum to the fundus. Gall bladder was taken out off through epigastric port. The patient was returned to a flat, supine position. The field was irrigated to ensure no bleeding or bile leakage. Drain were placed routinely in the operative bed (Morris pouch).

\section{Regarding to Para- umbilical hernia} repair: The camera was placed through the epigastric port and the fascial defect was closed primarily by interrupted polypropylene sutures, the skin was closed with absorbable subcuticular vicryl.

Operative time, postoperative complications, length of hospital stay were recorded. All patients were followed up on outpatient basis. They were scheduled for weekly visits in the first month, then every 2 weeks in the second month and once every month for the following 6 months, and finally 2 years postoperative for recurrence.

\section{Statistical Analysis:}

Results were tabulated and statistically analyzed by using a personal computer using MICROSOFT EXCEL 2016 and SPSS v. 21 (SPSS Inc., Chicago, IL, USA. Statistical analysis was done using. Descriptive: e.g. percentage (\%), mean and standard deviation. analytical: that includes t-test to compare quantitative data and Chi-square test and Fisher's exact test to compare quantitative data. A value of $\mathrm{P}$ less than 0.05 was considered statistically significant. 


\section{RESULTS}

A Retrospective study during the period from January 2017 to January 2019 and included Twenty-five patients at AlAzhar university hospitals with chronic calcular cholecystitis accompanied by Para-umbilical hernia who underwent laparoscopic cholecystectomy and Paraumbilical hernia repair simultaneously. All patients had been consented for laparoscopic cholecystectomy with paraumbilical hernia repair.

The mean age for cases with recurrence is $48 \pm 1.4$ and the mean age for cases without recurrence is $44 \pm 8.2$ with no statistically significant difference between the 2 groups. As regard to gender $50 \%$ are male and $50 \%$ are female for recurrence group, for cases without recurrence $8.7 \%$ are male and $91.3 \%$ are female with no statistically significant difference between the 2 groups. As regard to special habits 1 case is smoker for recurrence group, for cases without recurrence $4.3 \%$ are smoker, and $95.7 \%$ are with no special habits with statistically significant difference between the 2 groups. The mean BMI for cases with recurrence is $35.5 \pm 8.08$ and the mean BMI for cases without recurrence is $29.6 \pm 2.7$ with statistically significant difference between the 2 groups (Table 1).
All cases with recurrence have DM and hypertension while cases with no recurrence $87.3 \%$ have no comorbidities, 4.3\% have DM, $13 \%$ have hypertension and $4.3 \%$ have both DM and hypertension with statistically significant difference between the 2 groups. As regard to size of the defect for cases with recurrence and cases with no recurrence are $2.4 \pm .07$ and $1.3 \pm .4$ respectively with statistically significant difference between the 2 groups (Table 1).

The mean time of operation for cases with recurrence and cases with no recurrence are 90.00 and 49.5 \pm 7.2 minutes respectively with statistically significant difference between the 2 groups. Mean hospital stay for cases with recurrence and cases with no recurrence are $6 \pm 1$.4and $1.4 \pm 1.03$ days respectively with statistically significant difference between the 2 groups. Mean time to return to work for cases with recurrence and cases with no recurrence are $23 \pm 2.8$.4and $10.7 \pm 4.2$ days respectively with statistically significant difference between the 2 groups $50 \%$ of cases with recurrence have complication in form of haematoma and $50 \%$ have chest infection .cases without recurrence $95.7 \%$ have no complication and $4.3 \%$ have wound infection with statically significant difference between the 2 groups. 
Table (1): Socio-demographic data, relation between presence of comorbidities and size of the defect and recurrence, time of operation, hospital stay and return to work and recurrence, complication and recurrence

\begin{tabular}{|c|c|c|c|c|}
\hline \multicolumn{2}{|l|}{ Parameters } & Yes (2) & No (23) & $P$ value \\
\hline Age & & $48 \pm 1.4$ & $44 \pm 8.2$ & 0.515 \\
\hline \multirow{2}{*}{ Gender } & Males & $1(50 \%)$ & $2(8.7 \%)$ & \multirow{2}{*}{0.08} \\
\hline & Females & $1(50 \%)$ & $21(91.3 \%)$ & \\
\hline \multirow{2}{*}{ Special Habits } & Smoker & $1(50 \%)$ & $1(4.3 \%)$ & \multirow{2}{*}{0.022} \\
\hline & No & $1(50 \%)$ & $22(95.7 \%)$ & \\
\hline BMI & & $\begin{array}{c}35.5 \pm 8.0 \\
8\end{array}$ & $29.6 \pm 2.7$ & 0.019 \\
\hline \multirow{4}{*}{ Comorbidities } & No & 0 & $18(87.3 \%)$ & \multirow{4}{*}{0.001} \\
\hline & $\mathrm{DM}$ & 0 & $1(4.3 \%)$ & \\
\hline & Hypertension & 0 & $3(13 \%)$ & \\
\hline & $\begin{array}{c}\text { DM \& } \\
\text { hypertension }\end{array}$ & $2(100 \%)$ & $1(4.3 \%)$ & \\
\hline $\begin{array}{c}\text { Size of the } \\
\text { defect }\end{array}$ & & $2.4 \pm .07$ & $1.3 \pm .4$ & 0.001 \\
\hline \multicolumn{2}{|c|}{ Time of operation (minutes) } & 90.00 & $49.5 \pm 7.2$ & 0.001 \\
\hline \multicolumn{2}{|c|}{ Hospital stay (days) } & $6 \pm 1.4$ & $1.4 \pm 1.03$ & 0.000 \\
\hline \multicolumn{2}{|c|}{ Return to work (days) } & $23 \pm 2.8$ & $10.7 \pm 4.2$ & 0.001 \\
\hline \multirow{4}{*}{ Complications } & No & 0 & $22(95.7 \%)$ & \multirow{4}{*}{$<0001$} \\
\hline & Haematoma & $1(50 \%)$ & 0 & \\
\hline & $\begin{array}{l}\text { Wound } \\
\text { infection }\end{array}$ & 0 & $1(4.3 \%)$ & \\
\hline & $\begin{array}{c}\text { Chest } \\
\text { infection }\end{array}$ & $1(50 \%)$ & 0 & \\
\hline
\end{tabular}

There was moderate positive correlation between BMI and both time of operation and Length of hospital stay. There was strong positive correlation between Size of hernia and both time of operation and Length of hospital stay, and strong positive correlation between Comorbidity and both time of operation and Length of hospital stay (Table 2).

Table (2): The effect of BMI, Size of hernia\& Comorbidity on time of operation \& Length of hospital stay

\begin{tabular}{|c|c|c|c|}
\hline \multirow{2}{*}{ Parameters } & Values & $\begin{array}{c}\text { Time of } \\
\text { operation }\end{array}$ & $\begin{array}{c}\text { Length of } \\
\text { hospital stay }\end{array}$ \\
\hline \multirow{2}{*}{ BMI } & r- value & 0.433 & 0.487 \\
\cline { 2 - 4 } & P value & 0.031 & 0.014 \\
\hline \multirow{2}{*}{ Size of hernia } & r- value & 0.712 & 0.842 \\
\cline { 2 - 4 } & P value & 0.001 & 0.001 \\
\hline \multirow{2}{*}{ Comorbidity } & r- value & 0.856 & 0.418 \\
\cline { 2 - 4 } & P value & 0.001 & 0.038 \\
\hline
\end{tabular}

$81.8 \%$ of cases with no complication have no comorbidity and all cases with complication complaining of DM and hypertension (Table 3). 
Table (3): The effect of comorbidity disease on the complications

\begin{tabular}{|c|c|c|c|c|c|}
\hline $\begin{array}{c}\text { Complications } \\
\text { Comorbidity }\end{array}$ & No & Haematoma & $\begin{array}{c}\text { Wound } \\
\text { infection }\end{array}$ & $\begin{array}{c}\text { Chest } \\
\text { infection }\end{array}$ & P value \\
\cline { 1 - 5 } No & $18(81.8 \%)$ & 0 & 0 & 0 & \\
\cline { 1 - 5 } DM & $1(4.5 \%)$ & 0 & 0 & 0 & \multirow{2}{*}{0.003} \\
\cline { 1 - 4 } Dypertension & $3(13.6 \%)$ & 0 & 0 & 0 & \\
\hline
\end{tabular}

\section{DISCUSSION}

In this study, female to male incidence was 7.6:1 and this incidence was close to the work of Zoricic et al. (2013) which was 8.9:1. This dids not go with Bozkurl et al. (2012) who had female to male incidence about $2: 1$. This is probably due to the differences socioeconomic class and the social habits.

The mean age of the studied groups of patients in this work was 44.4 years. It was older than that of Bozkirll et al. (2012) (43.6 years) and Zoricic et al. (2013) (37.1 years). However, the elderly patients are not concerned with the problem of attractiveness.

In our study, the mean BMI for cases with recurrence was $35.5 \pm 8.08$ and the mean BMI for cases without recurrence was $29.6 \pm 2.7$ with statistically significant difference between the 2 groups.

Cases without recurrence $4.3 \%$ were smoker and $95.7 \%$ were with no special habits with statistically significant difference between the 2 groups.

Among the 25 patients had received simultaneous paraumbilical hernia repair with LC included in this study, 1 patient were diabetics, 3 patients were hypertensive and 1 patient were diabetics and hypertensive.

There was a strong positive correlation between comorbidity and both time of operation and length of hospital stay. This result was against Bozktrl et al. (2012) who mentioned that the comorbidity factors not affected the operative time but had negative impact on the both length of hospital stay and return to work.

In our study, $81.8 \%$ of cases were with no complication have no comorbidity and all cases with complication complaining of DM and hypertension. The shortening of hospital stay influences the reduction of costs, minimization of the patient discomfort and the reduction of complications.

Mean hospital stay for cases with recurrence and cases with no recurrence were $6 \pm 1.4$ and $1.4 \pm 1.03$ days respectively with statistically significant difference between the 2 groups.

Mean time to return to work for cases with recurrence and cases with no recurrence were $23 \pm 2.8 .4$ and $10.7 \pm 4.2$ days respectively with statistically significant difference between the 2 groups and the mean time of operation for cases with recurrence and cases with no recurrence were 90.00 and 49.5 \pm 7.2 minutes respectively with statistically significant difference between the 2 groups. This result agreed with Bozkirl et al. (2012) who mentioned that decreasing the length of hospital stay time may improve patient comfort and expedite recovery. Zoricic et al. (2013) showed no 
significant difference was detected in terms of mean length of hospital stay between the groups.

Recurrence rate is the most important factor in determining the optimal method for LC accompanied by simultaneous para-umbilical hernia repair. High recurrence rates have been reported after suture repair in many studies. Most surgeons currently prefer prosthetic mesh hernioplasty (Bozkirll et al., 2012 and Zoricic et al., 2013). In our study, 50\% of cases with recurrence have complication in form of haematoma and 50\% have chest infection. Cases without recurrence 95.7\% have no complications and $4.3 \%$ have wound infection with statistically significant difference between the 2 groups.

\section{CONCLUSION}

Para-umbilical hernia repair (PUHR) combined with $\mathrm{LC}$ result in longer time of operation, longer anesthesia, and risk of increased blood loss. However, it has the advantages of single hospital stay, single anesthesia exposure, less post-operative pain and morbidity, early return to work, better cosmesis and more convenient, efficacy and cost effective for the patient. However, the outcomes of the paraumbilical defect repair with mesh after laparoscopic surgeries appear to be better for either obese or non-obese patients than primary suture techniques in recurrence rates. Finally, PUHR combined with LC, kills two pathologies with one scope.

\section{REFERENCES}

1. Aslani N and Brown CJ (2010): Does mesh offer an advantage over tissue in the open repair of umbilical hernias? A systematic review and meta-analysis. Hernia, 14(5):455462.

2. Bozkırlı OP, Gundogdu RK, Basically H, Ersoy P, Turgay $M$ and Vulture $S$ (2012): The Significance of the Coexistence of Cholelithiasis and UmbilicalHernia in Laparoscopy Era. Gazi Med J., 23: 59 - 61.

3. Farquharson $M$, Hollingshead $J$ and Brendan M (2014): Farquharson's Textbook of Operative General Surgery. Pbl. CRC Press., p. 222.

4. Kulaçoğlu H (2015): Current options in umbilical hernia repair in adult patients. Turk. J. Surg., 31(3):157-162.

5. Lanaya $S$ and Manvi PN (2018): A clinical study and different modalities of management of ventral hernias. Int Surg J., 5(11):35893593.

6. Malik AM (2011): General Surgery Procedures, Advances in Laparoscopic Surgery part 1 Pp. 1-55.

7. Martis JJ, Rajeshwara KV, Shridhar MK, Janardhanan D and Sudarshan S (2011): Strangulated Richter's umbilical hernia-a case report. Indian J Surg., 73(6):455-457. 2011.

8. Savita KS, Khedkar I and Bhartia VK. (2010): Combined Procedures with Laparoscopic Cholecystectomy. Indian J Surg., $72(5)$ : $377-380$.

9. Zoricic I, Vukusic D, Rasic Z, Schwarz D and Sever M. (2013): Therapy of Umbilical Hernia during Laparoscopic Cholecystectomy. Coll Antropol., 37(3): 1003-1005. 


\section{تقييم إصلاح الفتق الجار سري المصاحب لعملية إستنصال

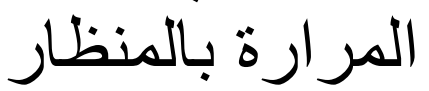

أحمد رضوان علي أحمد، سليم سعيد عبد الرحمن النمر، محمود عبد الهادي عبد العزيز قسم الجراحة العامة، كليـة الطـب، جامعة الأزهر

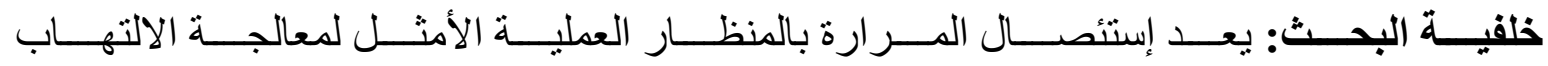

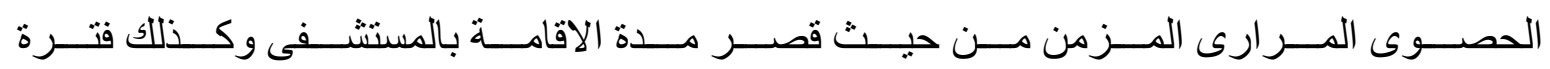

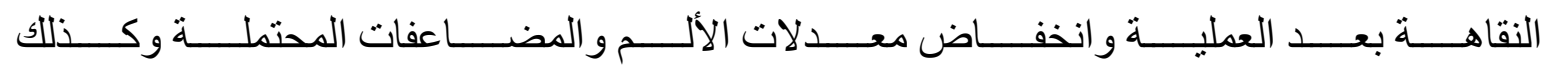

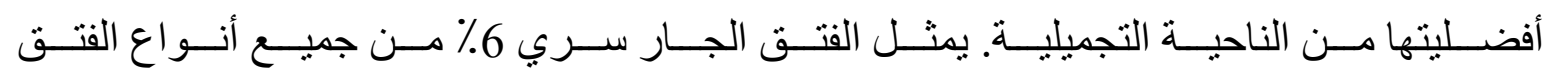

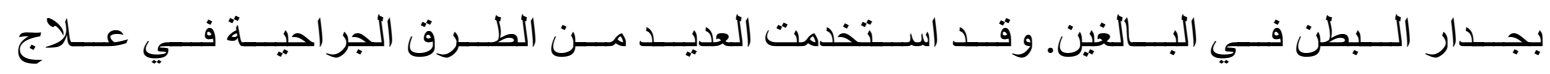

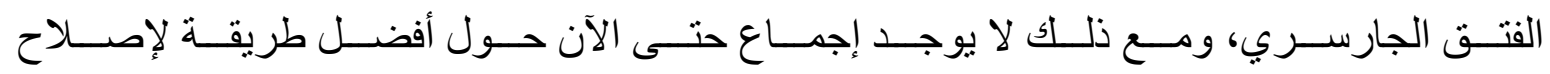

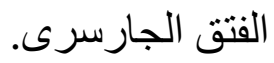

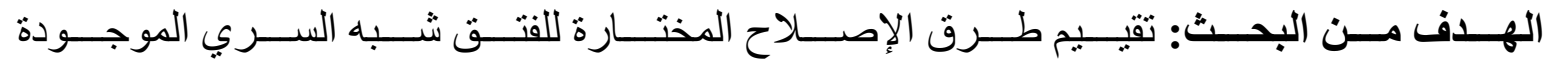

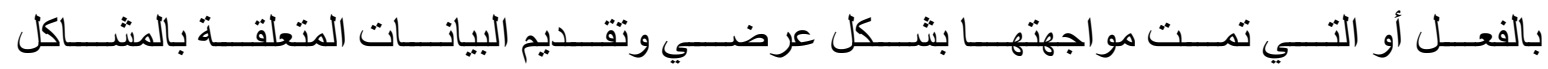

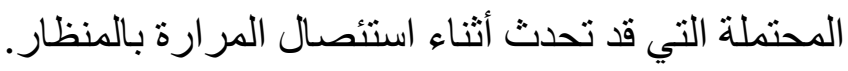

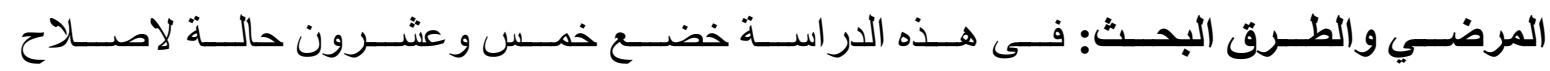

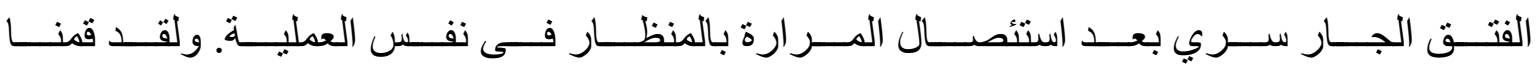

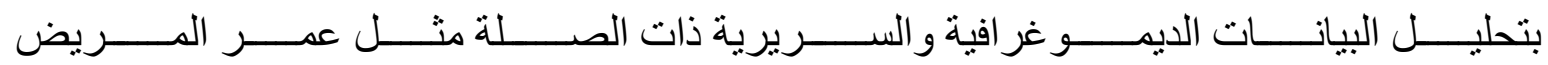

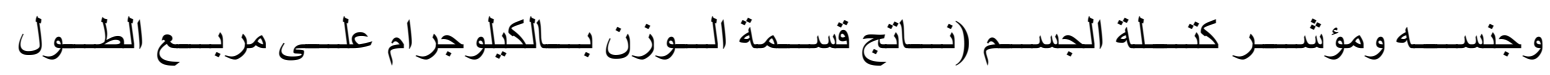

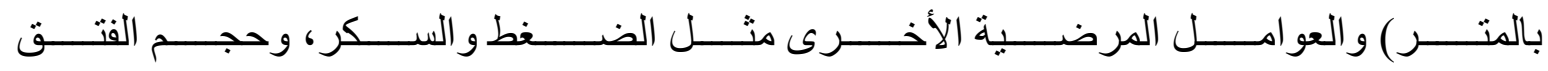

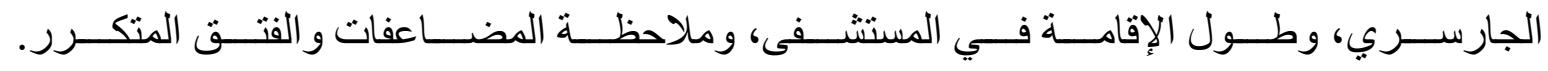

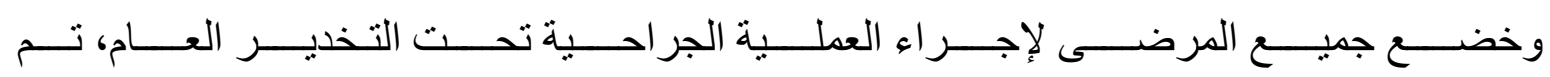

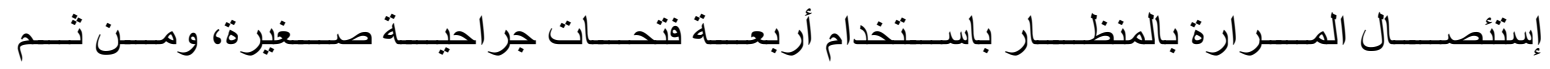

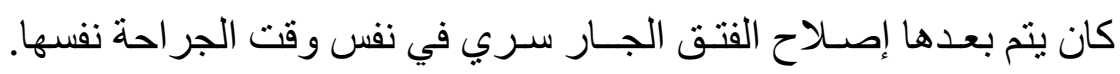

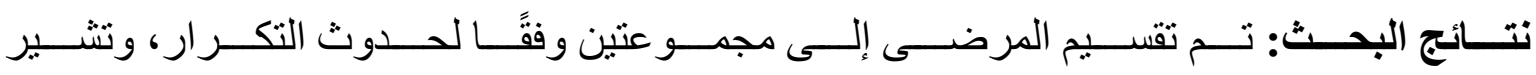

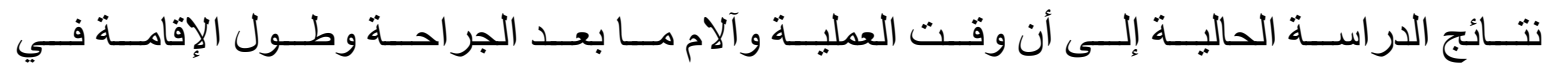

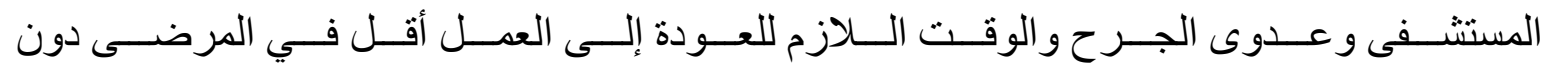




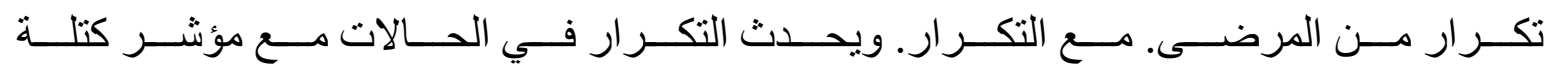

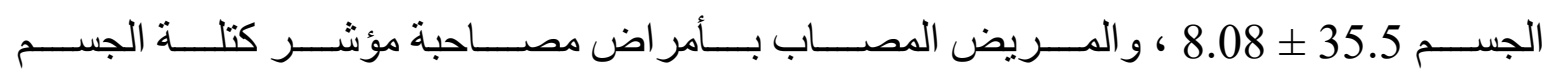

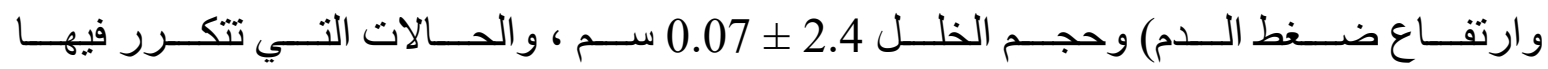

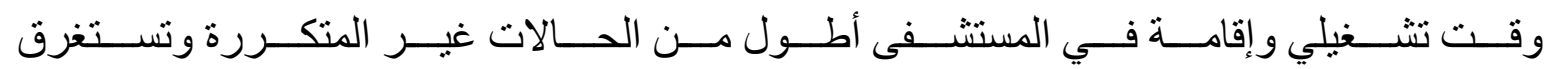

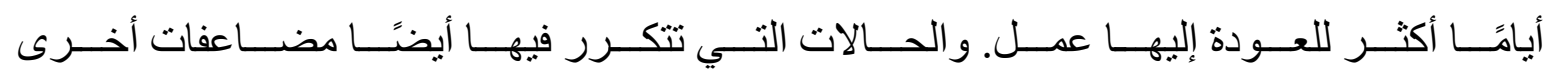

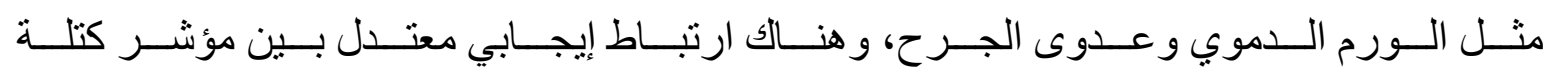

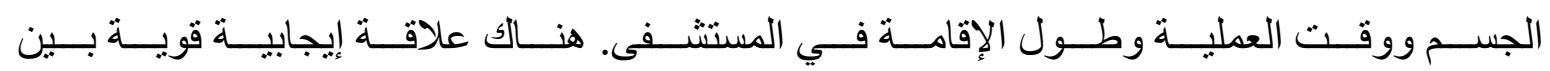

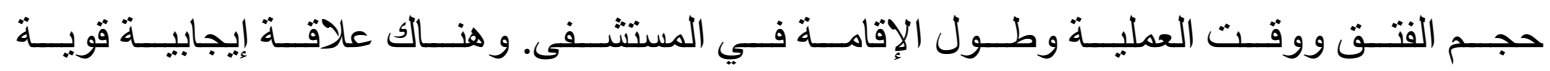
بين الاعتلال المشترك ووقت العملية وطول الإقامة في المستشفى.

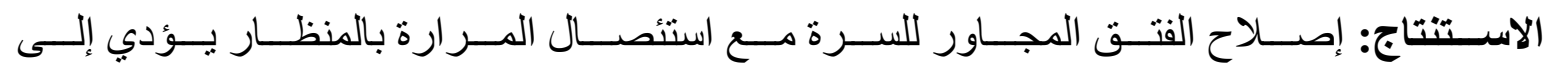

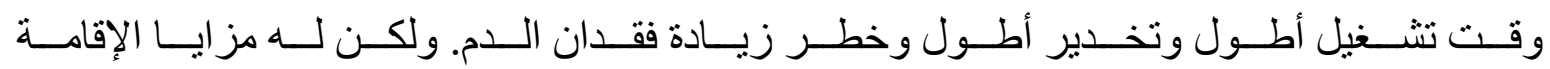

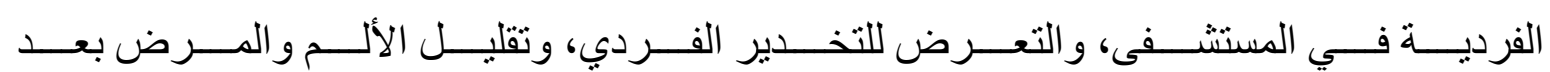

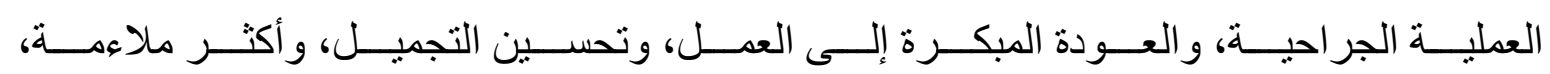

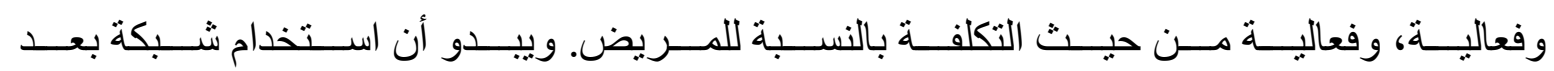

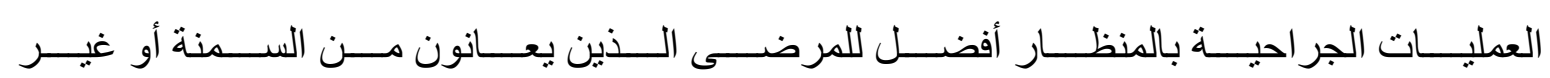
البدينين من تقنيات الخياطة الأولية في معدلات التكرار. 